\title{
THE MEASUREMENT OF SOUND ABSORPTION
}

\author{
By V. L. Chrisler and W. F. Snyder
}

\section{ABSTRACT}

This paper contains a brief discussion of the theory of reverberation measurements. The reverberation room is described and details are given as to the method of measuring sound absorption by means of the ear.

Methods are also described by which sound absorption can be determined from oscillograph records or by the use of an attenuator box and vacuum tube voltmeter.

\section{CONTENTS}

I. Introduction

Page

II. Methods available

957

III. Theory of the reverberation method

IV. Description of the reverberation room

V. Reverberation time for an acoustically dead room

VI. Source of sound

VII. Calibration of the reverberation room

VIII. Measurement of sound absorption coefficients by the ear method.-

IX. Measurement of sound absorption by instrumental methods.....

\section{INTRODUCTION}

Since radiobroadcasting and sound pictures have become important the problem of the acoustical treatment of auditoriums has been greatly emphasized. If the acoustic quality of a room is to be determined in advance and is not to be a matter of cut and try, it is necessary to know the sound-absorption coefficients of the materials used in the interior finish of the room.

For some years past the National Bureau of Standards has realized the importance of this problem and has carried on experiments to determine the best method of measuring these coefficients. It is the purpose of the present paper to describe the theory and the method now in use at the bureau for this purpose.

\section{METHODS AVAILABLE}

Two methods have been used for the determination of sound absorption coefficients - the reverberation method of W. C. Sabine ${ }^{1}$ and the tube method used by H. O. Taylor ${ }^{2}$ and others. At the time this work was undertaken by the bureau, now some six years ago, the tube method, being entirely instrumental, appeared to be more desirable than the reverberation method, which involved the use of the ear. In addition, the tube method had the advantage that small samples could be used. ${ }^{3}$ For these reasons this method was used for several years.

W. C. Sabine, Collected Papers on Acoustics, Harvard University Press; 1922.

H. O. Taylor, Phys. Rev., 2, p. 270; 1913.

3 Transmission and Absorption of Sound by Some Building Materials, B. S. Sci. Paper No. 526, p. 61. 
Notwithstanding the ease which such measurements could be conducted, it soon became evident that the results obtained by this method did not agree with those obtained by other observers using the reverberation method. In order to check and compare the two methods when carried out by the same observers, the National Bureau of Standards constructed a reverberation room which has now been in use for about two years.

As a result of this comparative study the bureau has discontinued the use of the tube method. The reasons for this change are discussed in an article entitled: "Absorption of Sound at Oblique Angles of Incidence," published in the Bureau of Standards Journal of Research for February, 1930.

\section{THEORY OF THE REVERBERATION METHOD}

W. C. Sabine,$^{4}$ the pioneer in the science of architectural acoustics, first developed and made use of a reverberation equation in making sound-absorption measurements. His discussion of the subject is spread at considerable length throughout his writings, and is not convenient for reference. A more compact and readable presentation is given by F. R. Watson ${ }^{5}$ in a publication which is, perhaps, not generally accessible. For this reason it may be well to give here a brief summary of the theory, following the line of Watson's discussion.

The equation for the decay of sound in a room, as developed by Jaeger ${ }^{6}$ is

where

$$
E=E_{0} e^{-K t}=\frac{4 A}{a v S} e^{-\frac{a v S t}{4 V}}
$$

$E=$ energy of sound per unit volume in the room at time $t$.

$A=$ rate of emission of energy from the source of sound.

$a=$ average coefficient of absorption.

$v=$ velocity of sound.

$V=$ volume of room.

$t=$ time at any instant after the source of sound is stopped.

$S=$ total area exposed to sound waves.

In the practice of the reverberation method as heretofore conducted, sound is produced from any suitable source, and suddenly cut off. The time is measured from the instant of the stopping of the source until the residual sound becomes inaudible.

The object of reverberation experiments is to determine the quantity, $a$, and this involves a knowledge of all other quantities in equation (1).

$E$ is determined, according to the precedure stated, by the threshold of hearing. This is assumed to be one physical unit for the normal ear, but may vary considerably from day to day with the same person. Over a short period of time, however, it may be fairly constant, though its absolute value is uncertain.

$A$ is a quantity rather difficult to determine absolutely, though the ratio of two values of $A$ may be determined quite accurately. This consideration, together with that in the preceding paragraph, sug-

i See footnote 1, p. 957. I F. R. Watson, The Absorption of Sound by Materials, Bull. No. 172, Univ. of Ill. Eng. Exp. Sta.;
Nov. 29, 1927 .

'Jaeger, "Zur Theorie des Nachballs," Akad. Wiss. Sitz. Ber., 120, 2a p. 613, 1911. 
gests the advisability of making two experiments in close succession with different values of $A$, and eliminating $E$. This is the procedure actually adopted in the calibration of the reverberation room. For output values of $A_{1}$ and $A_{1}^{\prime}$, equation (1) gives the following for an empty room of absorption coefficient $a_{1}$ and time of decay $t_{1}$.

Eliminating $E$ we get

$$
\left.\begin{array}{l}
E=\frac{4 A_{1}}{a_{1} v S} e^{-\frac{a_{1} v S t_{1}}{4 V}} \\
E=\frac{4 A_{1}^{\prime}}{a_{1} v S} e^{-\frac{a_{1} v S t_{1}^{\prime}}{4 V}}
\end{array}\right\}
$$

$$
a_{1} S=\frac{4 V \times 2.3 \log _{10}\left(\frac{A_{1}}{A^{\prime}}\right)}{v\left(t_{1}-t^{\prime}{ }_{1}\right)}
$$

the usual formula for calibration of a room by the reverberation-ear method.

Let us now introduce into the room a sample of material to be measured for sound absorption. Let its area be $S_{2}$ and its coefficient of absorption $a_{2}$. This leaves an area $S_{1}=S-S_{2}$ of the walls of the room with a coefficient $a_{1}$.

Equation (1) gives in this case

$$
E=\frac{4 A_{1}}{\left(a_{1} S_{1}+a_{2} S_{2}\right) v} e^{-\frac{\left(a_{1} S_{1}+a_{2} S_{2}\right) v t_{2}}{4 V}}
$$

By introducing the absorbent material in the room the initial intensity has been reduced from

$$
\frac{4 A_{1}}{a_{1} S v} \text { to } \frac{4 A_{1}}{\left(a_{1} S_{1}+a_{2} S_{2}\right) v}
$$

and therefore the measured time $t_{2}$ is a trifle shorter than it would be if the initial intensity were the same as that in the empty room. If it is now assumed that the initial intensity with the sample in room is increased to the initial intensity with the room empty, equation (4) becomes

$$
E=\frac{4 A_{1}}{a_{1} S v} e^{-\frac{\left(a_{1} S_{1}+a_{2} S_{2}\right) v t_{2}}{4 V}}
$$

From the first of equations (2) and (5)

$$
a_{2}=\frac{a_{1} S t_{1}-a_{1} S_{1} t^{\prime}}{S_{2} t^{\prime}}
$$

All of the quantities on the right have been measured except $t^{\prime}{ }_{2}$, but this can be computed in terms of $t_{2}$. From equations (4) and (5)

$$
t^{\prime}{ }_{2}=\frac{4 V}{\left(a_{1} S_{1}+a_{2} S_{2}\right)} 2.3 \log _{10}\left(\frac{a_{1} S_{1}+a_{2} S_{2}}{a_{1} S_{1}}\right)+t_{2}
$$

To solve this equation $a_{2}$ must be known, but a sufficiently exact value can be obtained by assuming $t^{\prime}{ }_{2}$ equal to $t_{2}$ and solving for $a_{2}$ 
from equation (6). This approximate value of $a_{2}$ is used in equation (7) and thus obtaining $t^{\prime}{ }_{2}$. The exact value of $a_{2}$ can now be computed from equation (6).

The foregoing theory applies to the method of reverberation as heretofore applied, using the threshold of the ear as a fixed point of reference. The modification of this method now in use at the National Bureau of Standards eliminates the ear as a factor, and measures the time required for the sound intensity to decay from one fixed level to another, starting from any initial intensity. The two levels of reference between which the time is measured are indicated instrumentally.

The foregoing equations for the ear method need but slight change to be applicable to this modified practice. If the levels of measurement are $E_{1}$ and $E_{2}$ equation (1) gives for the empty room:

$$
\left.\begin{array}{l}
E_{1}=E_{0} e^{-\frac{a_{1} v S S_{1}}{4 V}} \\
E_{2}=E_{0} e^{-\frac{a_{1} V S t_{2}}{4 V}}
\end{array}\right\}
$$

where $t_{1}$ and $t_{2}$ are now the times of passing the sound levels $E_{1}$ and $E_{2}$.

Eliminating $E_{0}$ and solving for $a_{1} S$, the total absorption of the empty room, we obtain:

$$
a_{1} S=\frac{4 V \times 2.3 \log _{10}\left(\frac{E_{1}}{E_{2}}\right)}{v\left(t_{2}-t_{1}\right)}
$$

After introducing the test sample we obtain by modifying similarly equation (4) and denoting the new times by $t_{1}{ }^{\prime}$ and $t_{2}{ }^{\prime}$ :

$$
a_{1} S_{1}+a_{2} S_{2}=\frac{4 V \times 2.3 \log _{10}\left(\frac{E_{1}}{E_{2}}\right)}{v\left(t^{1}{ }_{2}-t^{\prime}{ }_{1}\right)}
$$

from which $a_{2}$, the absorption coefficient of the sample may be calculated. It will be noticed that with the instrumental method it is not necessary to use two different output intensities, as $E_{0}$ is eliminated between the equations at the two levels $E_{1}$ and $E_{2}$.

\section{DESCRIPTION OF THE REVERBERATION ROOM}

In planning this room two features were taken into consideration, first, the period of reverberation should be long, and second, the walls should be good sound insulators to eliminate the disturbance from outside noises. Figure $1(a)$ and $(b)$ show the construction of the room. It is a rectangular room 25 by 30 by 20 feet. The inner and outer walls are of brick, 8 inches thick and there is an air space of 4 inches between the inner and outer walls. The ceiling is of reinforced concrete supported on the inner walls. The roof is composed of a second reinforced concrete slab placed about 16 inches above the ceiling, and carried on the outer wall. In this way there is no con- 
nection between the inner and outer shells except at the foundation, and the sound insulation is excellent. Except for sounds, which are transmitted by ground vibration, the four doors at the entrance
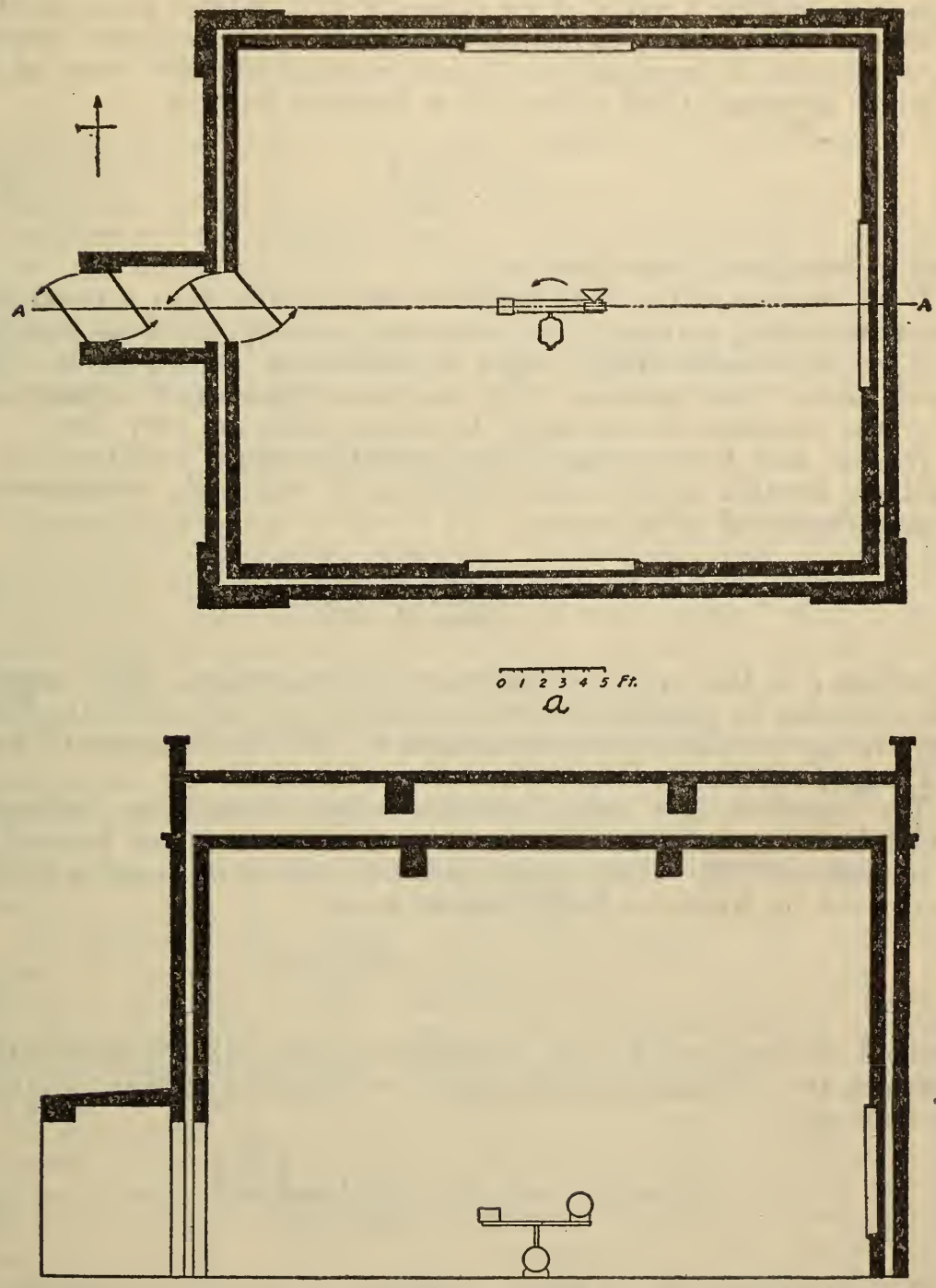

SECTION A-A

B

FIGURE 1.-Plan and cross section of reverberation room

(a) Plan; (b) Cross section.

permit more sound to pass through than any other portion of the building. It is seldom that enough sound comes through to disturb any measurements. 


\section{REVERBERATION TIME FOR AN ACOUSTICALLY DEAD ROOM}

W. C. Sabine ${ }^{7}$ defined the reverberation time of a room as the time required for a sound of an intensity of $1,000,000$ physical units or 60 decibels to decay to one physical unit or zero decibels. Setting $\mathrm{E}_{0}=1,000,000 \mathrm{E}$ in equation (1) and introducing the value of the velocity of sound, $1,088 \mathrm{ft}$./sec. for $v$, Sabine's formula

$$
T=\frac{0.05 \mathrm{~V}}{a S}
$$

may be obtained from equation (1).

If the reverberation time becomes very small, as in a room with much absorbing material, the value of $a$ as computed from equation (11) often exceeds unity, which is an absurd consequence. This result shows that equation (11), and also equation (1) from which (11) was obtained, do not apply to rooms which are very dead.

Norris $^{8}$ and Eyring ${ }^{9}$ have developed theoretical modifications of Sabine's formula to meet this objection, in which the reverberation time is expressed as follows:

$$
T=\frac{0.05 V}{-S \log _{\mathrm{e}}(1-a)}
$$

in which $a$ is the average coefficient of absorption. This removes the objection to the older formula, as $a$ becomes 1 when $T=0$. When the average coefficient does not exceed 0.1 the two formulas (11) and (12) agree within the limits of experimental error.

The question now arises whether sound absorption coefficients which have been obtained in the reverberation room can be used for $a$ in equation (12). If the equation for the decay of sound is written as derived by Norris or by Eyring we have:

$$
E=E_{0} e^{\frac{0 . S t \log _{e}(1-a)}{4 V}}
$$

instead of equation (1). If measurements are made instrumentally between two different intensity levels an equation similar to (9) can be derived:

$$
-S \log _{\mathrm{e}}(1-a)=\frac{4 V \times 2.3 \log _{10}\left(\frac{E_{1}}{E_{2}}\right)}{v\left(t_{2}-t_{1}\right)}
$$

and the average $a$ for the empty room can be solved for as before. If this average $a$ is defined as

$$
\frac{a_{1} S_{1}+a_{2} S_{2}+\ldots}{S}
$$

i See footnote 1, p. 957 .

- R. F. Norris, of the Burgess Laboratories, Madison, Wis., private communication, as yet unpublished

- Carl F. Eyring, Reverberation Time in Dead Rooms, Reprint B-465, Bell Telephone Laboratories. 
where $S$ is the total area and $S_{1}, S_{2}$, etc., the areas of the materials having coefficients $a_{1}, a_{2}$, etc., then when a sample is placed in the room equation (14) becomes:

$$
-S \log _{\mathrm{e}}\left(1-\frac{a_{1} S_{1}+a_{2} S_{2}}{S}\right)=\frac{4 V \times 2.3 \log _{10}\left(\frac{E_{1}}{E_{2}}\right)}{v\left(t_{2}-t_{1}\right)}
$$

and $a_{2}$ can be solved for, as the other quantities are all known or can be measured.

The largest absorption coefficient which has been measured so far in the bureau's reverberation room is 0.94 as calculated by equation (9) or 0.91 by equation (15). The average coefficient for the whole room in this case was a trifle less than 0.04 . It is therefore safe to say that in none of the measurements to date, which seldom rise above 0.50 , has the reverberation room been dead enough to cause the result to depend appreciably on the formula used.

\section{SOURCE OF SOUND}

In selecting a source of sound two features are desirable, first, that a pure note of any desired pitch can be produced, and second, that the intensity can be easily changed through a wide range. After considering various sources of sound it was decided that a loudspeaker with a suitable electric oscillator was the most satisfactory method of accomplishing the above results.

After deciding upon a loud-speaker as a source of sound it was found that care must be taken in the choice of the type, as many speakers do not faithfully reproduce what is put into them. The cone apparently breaks up into segments, and overtones are produced which are not present as harmonics in the current supplied to the loud-speaker. It is also desirable that the acoustical output be directly proportional to the electrical input.

The speaker finally chosen was of the dynamic type, and when tested for the above properties was found to be satisfactory. The quality of the tone was checked by receiving the sound electrically and then amplifying with a high-grade resistance-coupled amplifier and examining the output on an oscillograph. On viewing the image in a rotating mirror no appreciable harmonics were detected.

To determine the relation between the electric input and the acoustical output of the source of sound, the source (a loud-speaker was mounted in the room used for transmission measurements. To obtain as uniform a distribution of sound as possible the loud-spealker was rotated and a narrow band of frequencies used instead of a single frequency. Three different types of electric receivers were used, (1) a special telephone receiver made by the Bell Telephone Laboratories, (2) a loud-speaker in which a movable coil attached to a metallic diaphragm moved in an electromagnetic field, and (3) a condenser microphone. Several different strengths of current were applied to the source, and relative intensity was measured with the different receivers. In each case the square root of the sound intensity was found to be proportional to the current in the source. The results for one frequency are give in Figure 2. 
In the first work a Vreeland oscillator was used as a source. The output of this oscillator was remarkably free from harmonics, but there were some undesirable features. A beat-frequency oscillator has been developed which is much more satisfactory. Figure 3 gives the wiring diagram. There are a few points about this circuit which may be of general interest although the ideas are not original.10 To prevent the two oscillating circuits from being drawn together in frequency when the beat frequency is low, a stage of radioamplification has been added to each oscillator. With this loose coupling it is possible to go as low as 10 cycles without appreciable distortion. The freedom from distortion is also partly due to the careful shielding between the different sections of the oscillator. Special fixed condensers were used in the tuned circuits to prevent frequency drift with temperature changes. The results have been excellent. Over a period of an hour or more there has never been any appreciable frequency drift. The a. c. output voltage is about 4 volts, and approximately constant from 50 to 6,000 cycles.

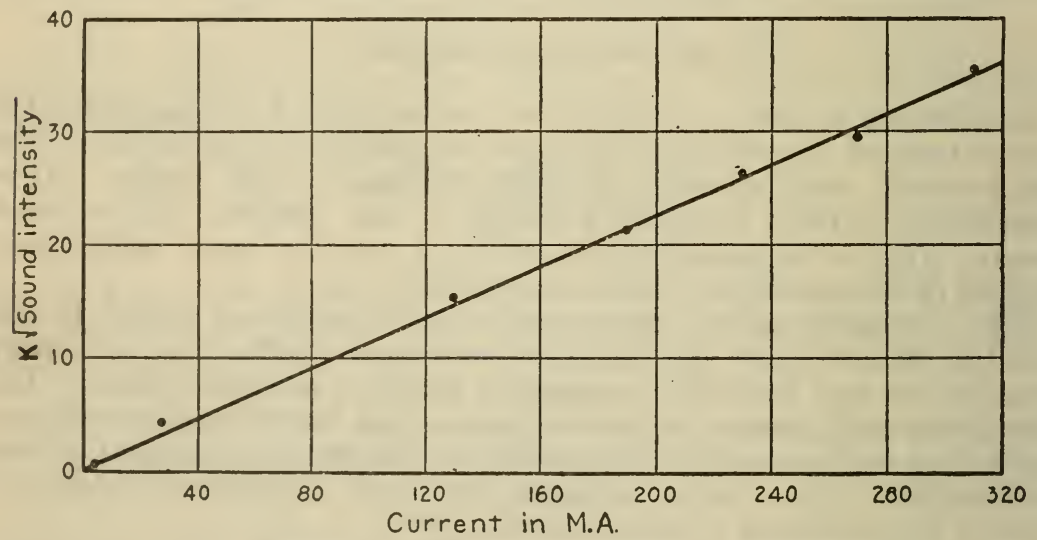

FIGURE 2.-Intensity calibration of loud-speaker

For some work it is desirable to use a tone which varies through a narrow band. To accomplish this, a small rotating variable condenser is placed externally in parallel with the condensers in the tuned circuit of one of the oscillators. The width of this band can be controlled by proper adjustment of the rotating condenser.

To obtain sufficient energy for the loud-speaker the output of the beat-frequency oscillator is fed into a high-grade resistance-coupled amplifier, the output of this amplifier going to the loud-speaker.

\section{CALIBRATION OF THE REVERBERATION ROOM}

The reverberation room was first calibrated by the ear method, using equation (3). 'This is essentially the plan used by Sabine in his four-organ-pipe experiment. The principal difference in the two experiments is that by using a loud-speaker it is easy to vary the intensity ratio by as much as 1,600 instead of being limited to 4 .

10 G. F. Lampkin, Radio Broadenst, 13, No.3, p. 157; July, 1928. Cohen, Aldridge and West, J. Inst. Radio Engrs. (London), 64, 1023; 1926. 
Five stations for measurement were chosen, arranged in an irregular manner around the floor. The observer was seated in a box for the purpose of eliminating the absorption of his clothing.

To obtain the total absorption of the room at any given frequency 10 observations were made for each intensity. Six intensities were

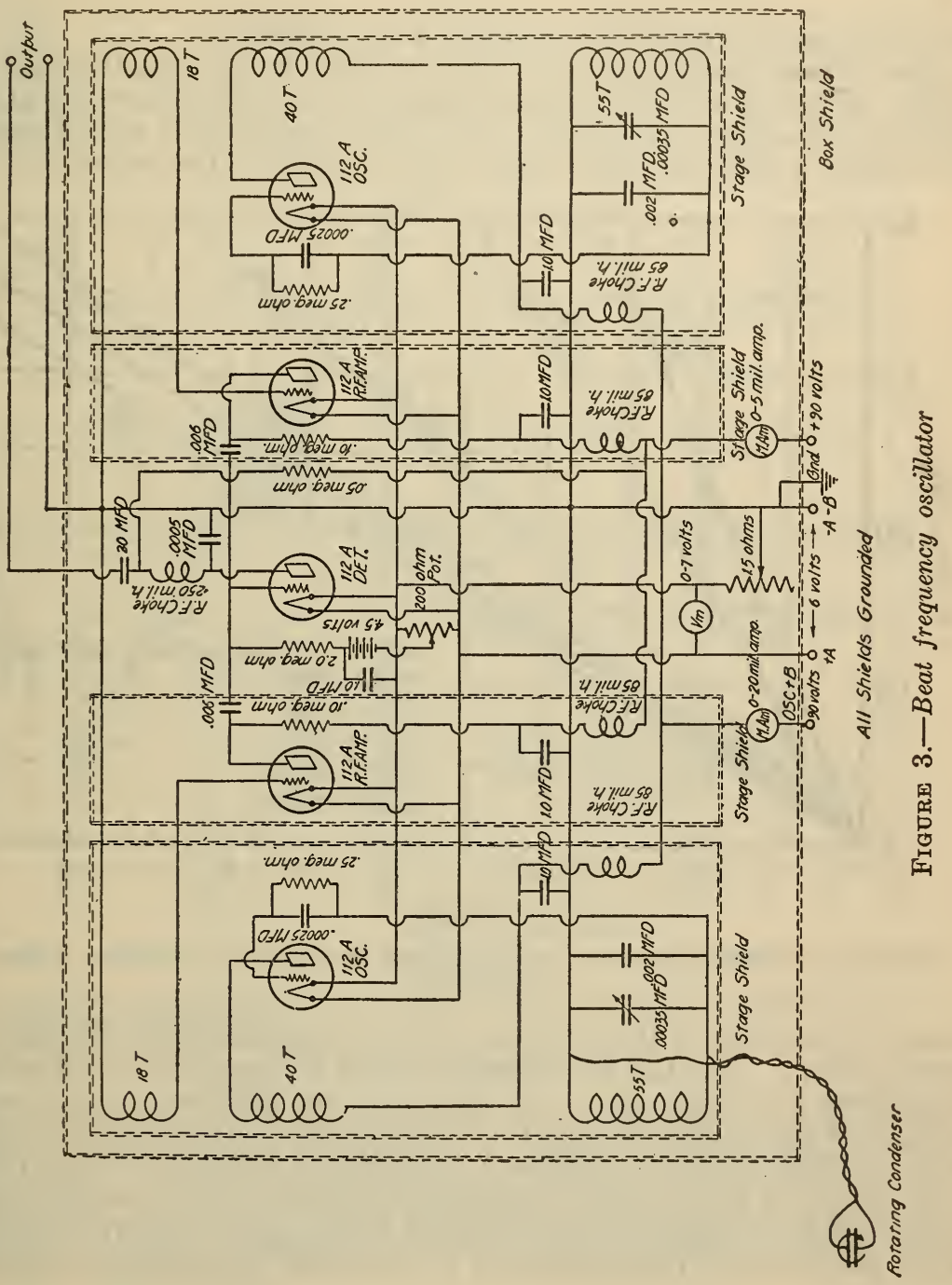

taken, the minimum corresponding to a 4 milliampere input to the loud-speaker and the maximum to 170 milliamperes input. This required the observer to make 300 observations to obtain the total absorption of the empty room for a single frequency. The figure finally used was the average of those obtained by two observers. The results of some of these observations are shown in Figure 4. 
As the acoustical output of the loud-speaker is proportional to the square of the current, $E=K C^{2}$ and equation (9) becomes

$$
a_{1} S=\frac{120.9 \times 2 \log _{10}\left(\frac{C}{C_{1}}\right)}{t-t_{1}}
$$

when the proper values are substituted for $V$ and $v$.

After a large number of observations it was found that the time of decay, except at 128 cycles, was independent of the observer's position when the room was empty. In a few cases when a very absorbent sample was in the room there was a change in the period with a

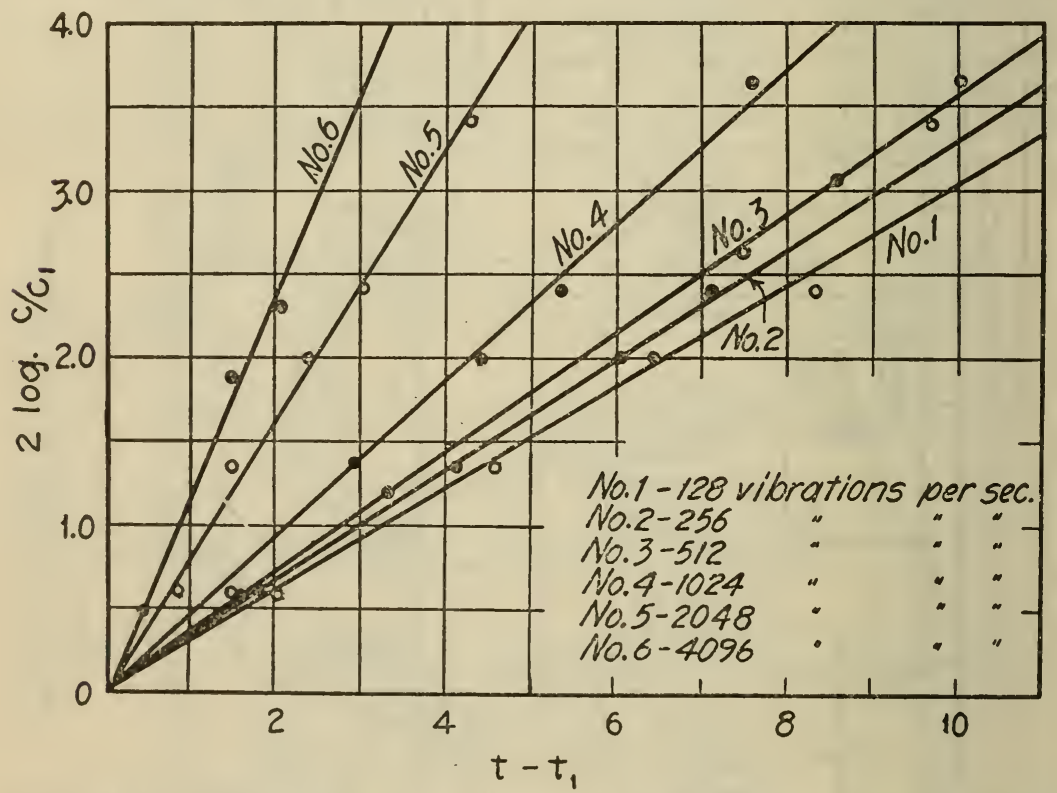

Figure 4.-Relation between period of decay of sound and logarithm of current ratio

change in position of the observer which was greater than the experimental error. This has not exceeded 0.2 of a second, the total period being from 6 to 8 seconds. At 128 cycles the difference under some conditions was somewhat larger. That the difference in period should be as small as possible at different positions in the room is of importance, as one of the conditions imposed in deriving the formula is that there shall be a uniform distribution of the sound energy at all times. If the period is not independent of the position this condition has not been met.

\section{MEASUREMENT OF SOUND ABSORPTION COEFFICIENTS BY THE EAR METHOD}

The usual method of determining the absorption coefficient of any material has been to determine the period of decay with the room empty and agrain with the test sample in the room. From equations $(3),(5)$, and (6) the coefficients of the material could be computed 
To determine experimentally whether equation (6) gave the proper correction a few sets of measurements were made on different samples at 512 cycles. The results are shown in Figure 5. The curves 1 and 3 intersect at $t=0$, as they should.

For an ordinary determination of time of decay 10 observations were made at each frequency, and this series repeated at a second station. The intensity was then changed and the whole set repeated. As a rule, the results from these measurements were in fair agreement. As a check determination, all of the measurements were repeated on a second day. This required not less than 960 observations to be made to determine the absorption coefficients at six frequencies for a single

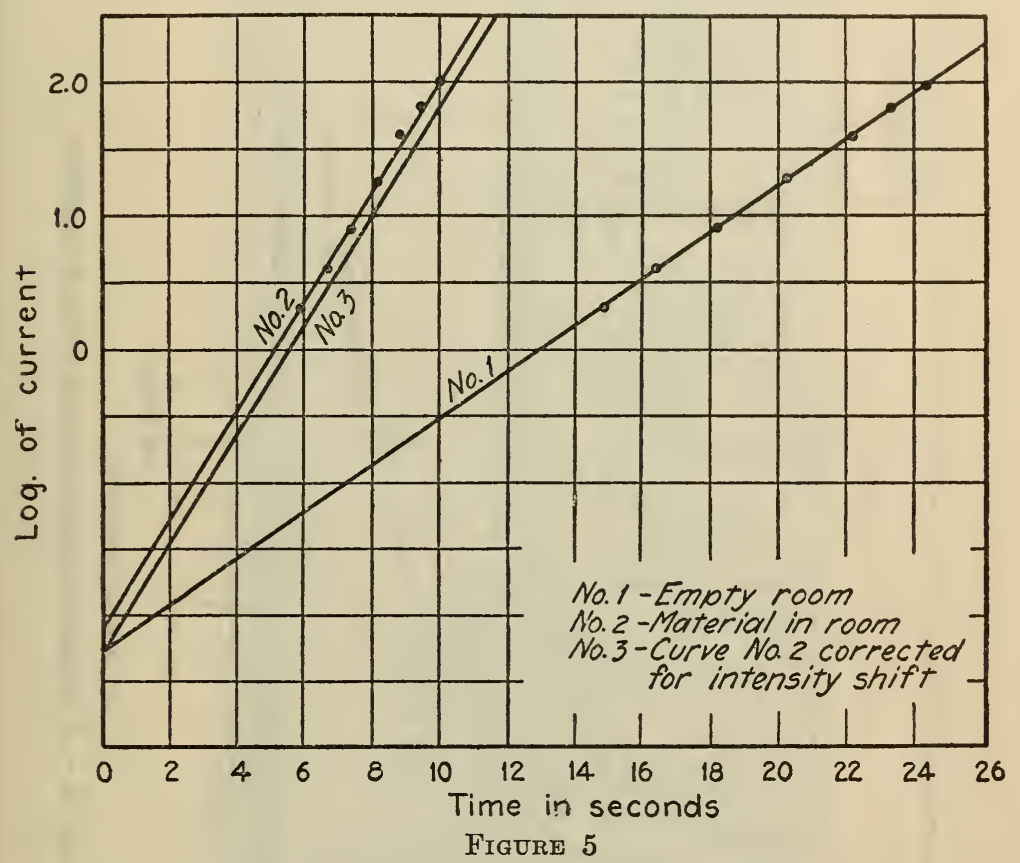

1. Relation between logarithm of current in loud-speaker and period of decay of sound without absorbing material in room.

2. Same with absorbing material in room.

3. Curve 2 corrected for intensity shift due to absorbing material.

sample, the equivalent of two days of strenuous work for a single observer. If the two sets did not check, additional measurements had to be made. As this involved entirely too much work it soon became evident that it was desirable to develop a method in which a single observation was more accurate, so that it would not be necessary to take so many measurements.

\section{MEASUREMENT OF SOUND ABSORPTION BY INSTRU- MENTAL METHODS}

From the results of ear measurements it was thought that the sound intensity decayed in a nearly uniform manner, and this suggested that an oscillograph record might be taken from which the absorption coefficient might be computed. 
To obtain these recorảs it was necessary to use a suitable electrical receiver and amplifier. Various electrical receivers were used, but a condenser microphone proved the most satisfactory. To obtain the necessary undistorted energy to operate the oscillograph a 5-stage

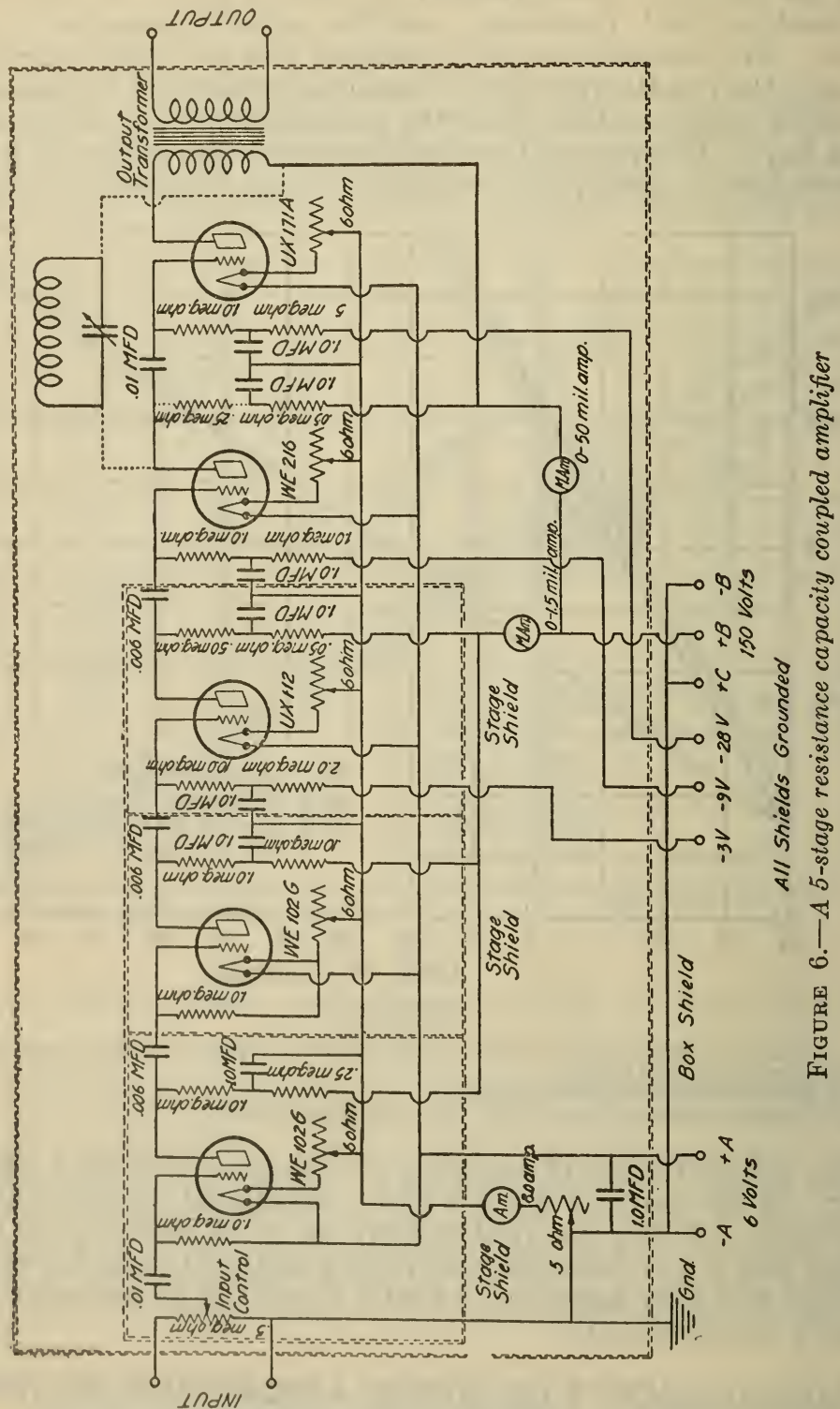

resistance-coupled amplifier was built. $\Lambda$ diagram of this circuit is shown in Figure 6 . The next to the last stage contains a tuned circuit which can be used when desired. This aids in eliminating all sounds except those for which the circuit is tuned. 


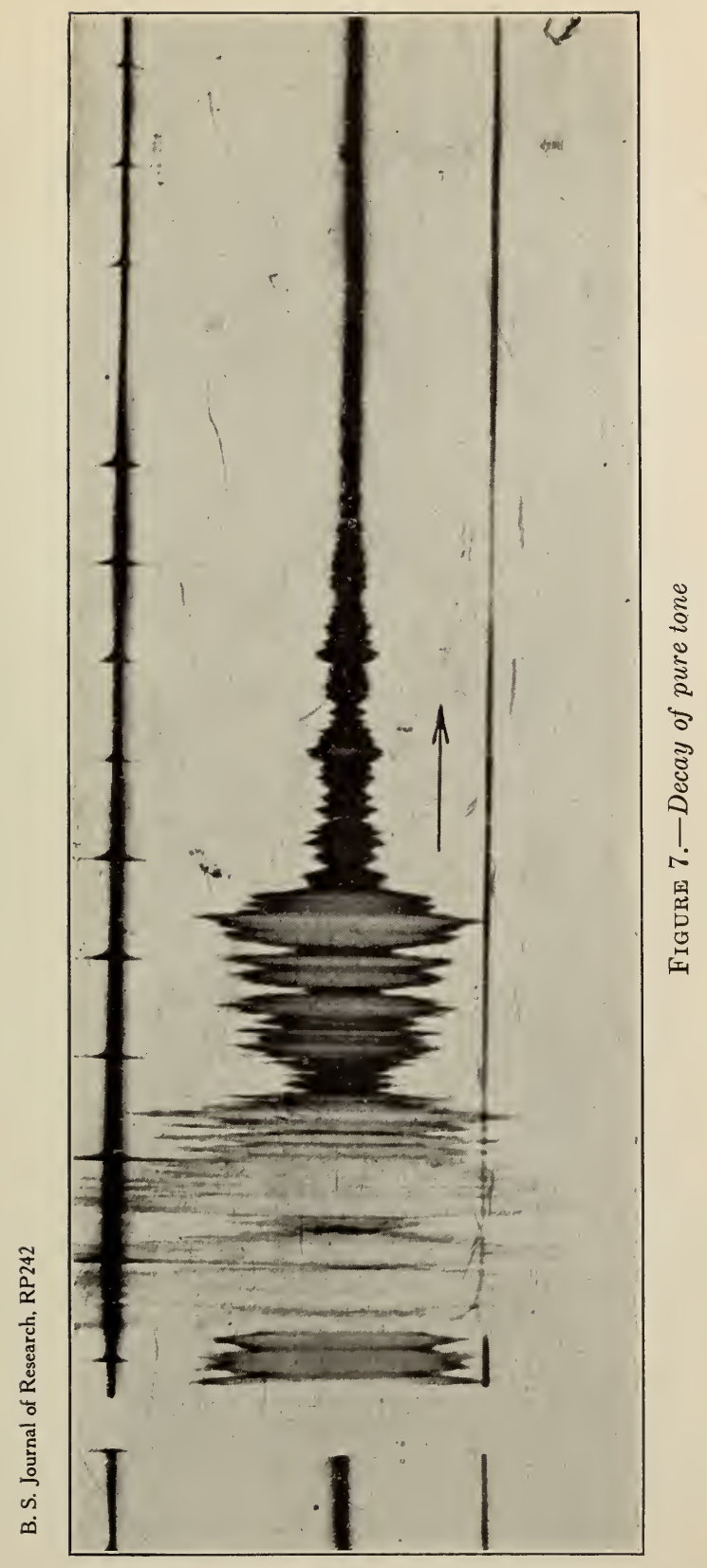




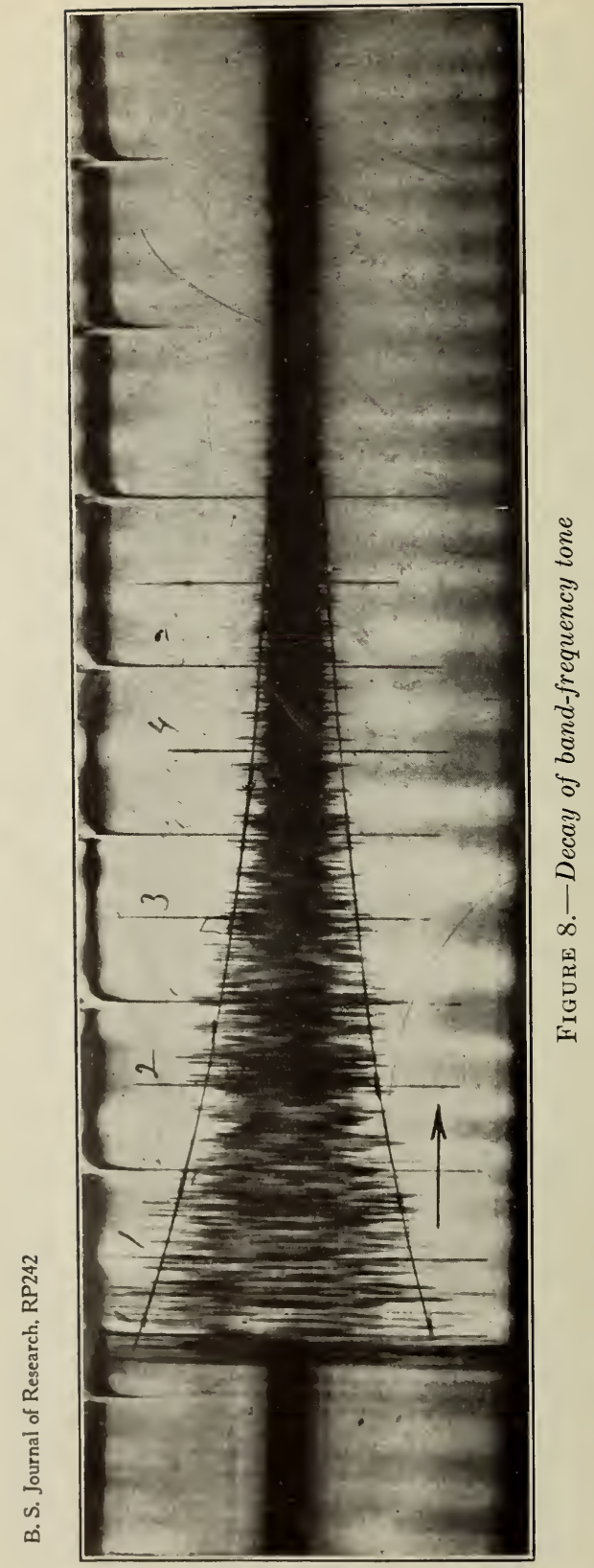


Theoretically the curves recorded photographically should show a logarithmic decay for the sound energy; actually they do not always do so. Figure 7 illustrates the decay of a low-pitched note of a single frequency.

After obtaining a few films like this it became evident that steps must be taken to obtain a distribution of sound which was more nearly uniform at each instant. To accomplish this the source of sound was rotated and instead of using a single frequency, a band was used, its width being about 10 per cent of the average frequency. For example, at 512 cycles the lower end of the band was about 25 cycles below 512 and the upper about 25 cycles above. Jointly these two procedures produced a much more uniform distribution. Figure 8 shows one of the films obtained under these conditions. In this case it is possible to draw an envelope to the curve, and by measuring its ordinates at a number of values of the time the rate of

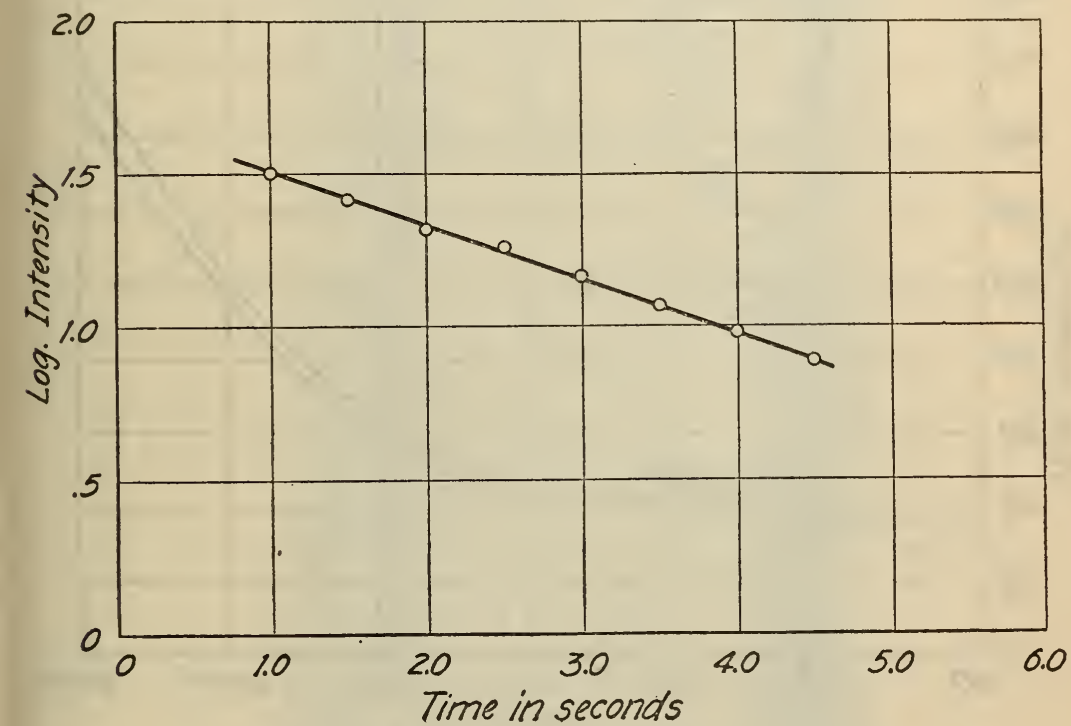

FigURE 9.-Rate of decay from oscillogram record

decay can be computed. Knowing the rate of decay, the absorption can be computed from equation (3). To show that the rate of decay was logarithmic the ordinates of the above film have been squared and their logarithms plotted against the time. The results are shown in Figure 9.

In this manner a calibration of the reverberation room was made, which is shown in Figure 10 along with the calibration previously described, made by ear. There was a little additional equipment in the room when the calibration was made by the oscillograph, but it is doubtful if this would account for the difference of four or five units of absorption shown in the curves.

An attempt was also made to eliminate some of the irregularities by rectifying the current and using large chokes and condensers in the electrical circuit. Figure 11 shows the result. It is quite evident 
there is no improvement; in fact, it would be easier to draw an envelope to Figure 8.

The results shown in Figure 10 are the average of a considerable number of films. It was found that the result of a single film was not dependable. The variation is probably caused by a gradual drift of the sound pattern in the room past the receiver as the sound dies away. It might be considered that two things are taking place. First, the sound is decaying, and second, the drift of the sound pattern either tends to increase or decrease the rate of decay. If the drift is such as to increase the rate of decay the apparent absorption is too large, while if the drift decreases the rate of decay, the apparent absorption is too small. If a sufficient number of films were taken this variation would probably average out, but the time required for this is greater than that required to take the measurements by ear.

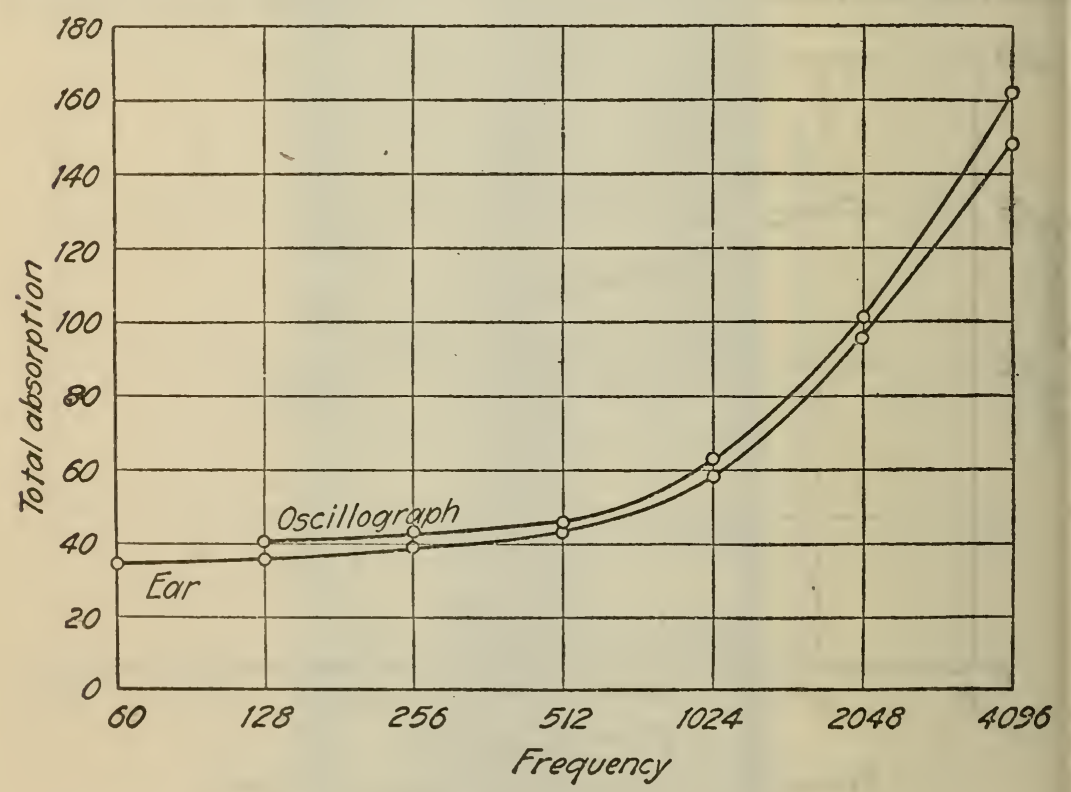

FiguRe 10.-Calibration of reverberation room

As the oscillograph gives a permanent record, which is always desirable, it seemed best to make further efforts to secure satisfactory records of the sound decay. If a record could be made through a sufficiently long-time interval it seemed possible that the drift of the sound pattern might be averaged out. To accomplish this it was necessary to change the amount of amplification while taking the record. This was accomplished by the use of an attenuator in the amplifier circuit. Figure 12 shows how such a record looks. By drawing envelopes to these two decay curves and then drawing parallel lines as shown we can determine the time that is required for the sound to decay a definite amount. The amount of this decay can be determined by using a calibrated attenuator box. The absorption can then be calculated as before from equation (9). 


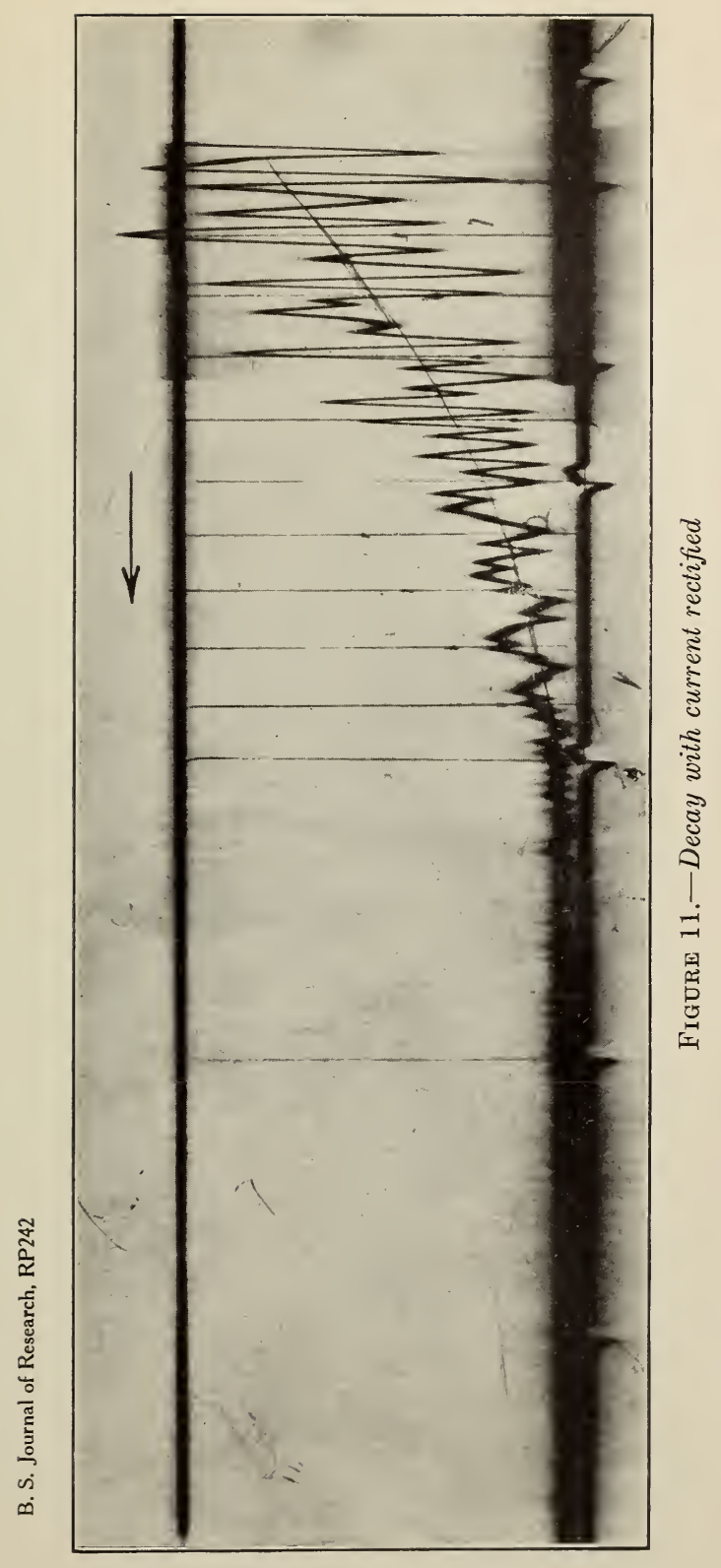




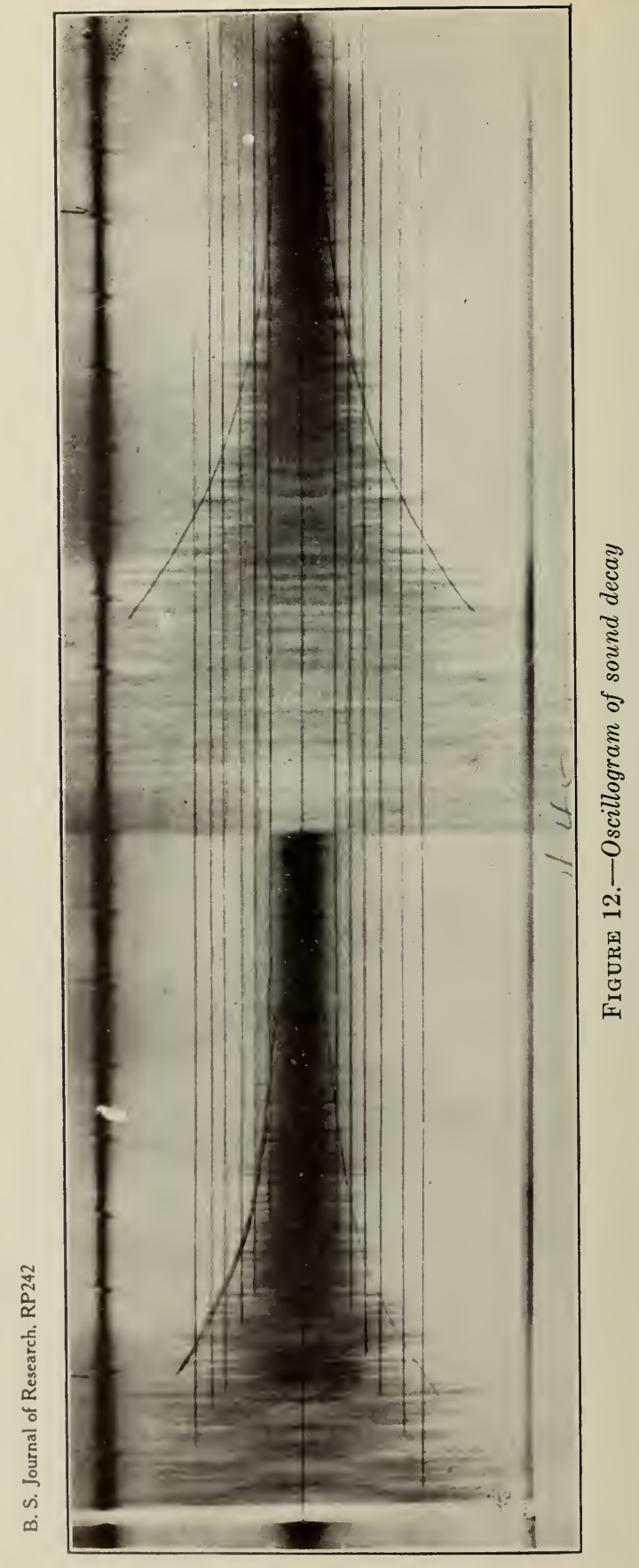


This gives us a method which is very similar to the classical reverberation method. The principal difference lies in the fact that we no longer use the threshold of audibility, but determine the time of decay for a measured change of intensity. A large number of records have been made in this manner, but the results are somewhat disappointing when used to determine the absorption coefficients of materials. It was hoped that the time of decay of a given sound through a given change of intensity would be constant provided other conditions remained constant. It was found, however, that the time was not a constant due probably to the drift of the sound pattern. Variations were found which were somewhat smaller than those obtained by a good observer using a stop watch to measure the time of decay of the sound, as the error due to the personal element was eliminated. As a result of this it was found necessary to take a number of films and use the average if satisfactory results were to be obtained when measuring the sound absorption coefficient of samples of material.

As it is a slow process to take oscillograph records and work them up, the oscillograph has finally been abandoned and the process slightly modified. The oscillograph method as described consisted

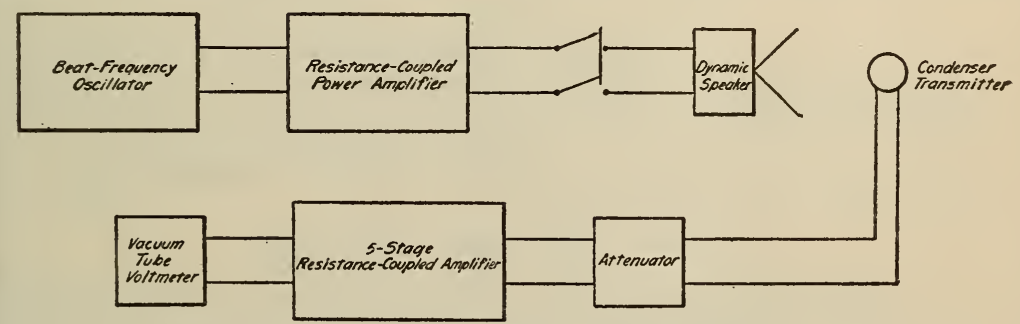

FIGURE 13.-Schematic diagram of electrical apparatus

Sketch shows entire arrangement

essentially in determining the length of time required for a sound to decay by a given amount. This can also be done by substituting a rectifying tube and microammeter.

The process of measurement is as follows. After the source has been on for a second or so the sound is cut off. Some arbitrary point is taken on the microammeter scale and as the snund dies away the microammeter needle passes this point. At that instant a timer is started by the observer and a helper changes the attenuator box a given amount so as to decrease the attenuation. The current is thus increased in the microammeter. As the sound continues to decay the needle again drops back through the same point and at this instant the timer is stopped. Knowing the change in attenuation and the time, the absorption can be computed from equation (10).

The measurements can be made much faster in this manner, although the accuracy of determining the time interval by a single measurement is not as good as that obtained from a single film taken with the oscillograph.

To obtain satisfactory results it is necessary to make a large number of measurements, just as when the ear is used as a measuring instrument, but it is believed that the accuracy of measurement is somewhat greater.

$$
11295^{\circ}-30-13
$$


The results of some of the measurements are given in Table 1.

TABLE 1.

\begin{tabular}{|c|c|c|c|c|c|c|c|}
\hline \multirow{2}{*}{ Sample No. } & \multirow{2}{*}{ Method } & \multicolumn{6}{|c|}{ Absorption coefficients at frequencies- } \\
\hline & & 128 & 256 & 512 & 1,024 & 2,048 & 4,096 \\
\hline $1 \ldots$ & $\left\{\begin{array}{l}\text { Instrument } \\
\text { Oscillograph } \\
\text { Ear }\end{array}\right.$ & $\begin{array}{l}0.14 \\
.16 \\
.16\end{array}$ & $\begin{array}{r}0.27 \\
.30 \\
.28\end{array}$ & $\begin{array}{r}0.53 \\
.53 \\
.52\end{array}$ & $\begin{array}{r}0.62 \\
.63 \\
.66\end{array}$ & 0.60 & $\begin{array}{r}0.54 \\
.42\end{array}$ \\
\hline 2 & $\left\{\begin{array}{l}\text { Instrument... } \\
\text { Ear.......... }\end{array}\right.$ & $\begin{array}{l}.08 \\
.10\end{array}$ & $\begin{array}{l}.13 \\
.14\end{array}$ & .25 & .54 & $\begin{array}{l}.67 \\
.67\end{array}$ & $\begin{array}{l}.42 \\
.40\end{array}$ \\
\hline 3... & $\left\{\begin{array}{l}\text { Instrument. } \\
\text { Ear }\end{array}\right.$ & .19 & .34 & .63 & $\begin{array}{l}.75 \\
.75\end{array}$ & .77 & .52 \\
\hline
\end{tabular}

Washington, July 8, 1930. 